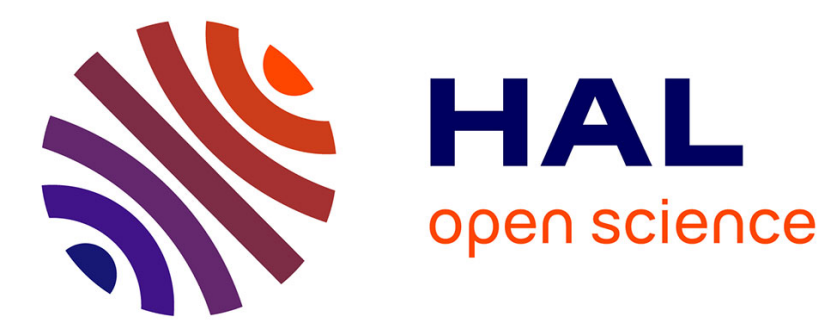

\title{
Fourier-modal methods applied to waveguide computational problems
}

Philippe Lalanne, Eric Silberstein

\section{To cite this version:}

Philippe Lalanne, Eric Silberstein. Fourier-modal methods applied to waveguide computational problems. Optics Letters, 2000, 25 (15), pp.1092-1094. hal-00867919

HAL Id: hal-00867919

https://hal-iogs.archives-ouvertes.fr/hal-00867919

Submitted on 30 Sep 2013

HAL is a multi-disciplinary open access archive for the deposit and dissemination of scientific research documents, whether they are published or not. The documents may come from teaching and research institutions in France or abroad, or from public or private research centers.
L'archive ouverte pluridisciplinaire HAL, est destinée au dépôt et à la diffusion de documents scientifiques de niveau recherche, publiés ou non, émanant des établissements d'enseignement et de recherche français ou étrangers, des laboratoires publics ou privés. 


\title{
Fourier-modal methods applied to waveguide computational problems
}

\author{
Philippe Lalanne and Eric Silberstein
}

Laboratoire Charles Fabry de l'Institut d'Optique, Centre National de la Recherche Scientifique, B.P. 147, F-91403 Orsay Cedex, France

\author{
Received February 4, 2000
}

Rigorous coupled-wave analysis (also called the Fourier-modal method) is an efficient tool for the numerical analysis of grating diffraction problems. We show that, with only a few modifications, this method can be used efficiently for the numerical analysis of aperiodic diffraction problems, including photonic crystal waveguides, Bragg mirrors, and grating couplers. We thus extend the domain of applications of grating theories. (C) 2000 Optical Society of America

OCIS codes: $050.1960,050.1950,130.2790,230.7390$.

Rigorous coupled-wave analysis ${ }^{1}$ (RCWA) is a versatile tool for modeling grating diffraction problems. It is a frequency-domain method based on computations of the grating modes as eigenvectors and on Fourier expansions for the permittivity and for the electromagnetic fields inside the grating region. With $\mathbf{S}$ or R-matrix propagation algorithms, ${ }^{2}$ RCWA can efficiently solve almost all one-dimensional grating diffraction problems with present desktop computers. In this Letter we provide numerical evidence that, with a few minor modifications, RCWA can also be used for modeling aperiodic structures, especially waveguide structures such as photonic crystal waveguides, Bragg mirrors, and grating couplers. The following discussion is restricted to planar waveguide geometries for $\mathrm{TE}$ and TM polarizations. The reader is assumed to be familiar with the RCWA method, ${ }^{1}$ with its enhanced version $^{3}$ for TM polarization, and with the S-matrix propagation algorithm ${ }^{2}$ used for analyzing multilayer structures.

Figure 1 shows one possible waveguide geometry that will be used here to illustrate the method. This geometry is the simplest realization of a photonic bandgap structure; it was used recently as a thirdorder mirror in a short-cavity AlGaAs semiconductor laser ${ }^{4}$ operating at $975 \mathrm{~nm}$. For the following discussion the corrugation profile is not necessarily binary or periodic in the $z$ direction. Both metallic and dielectric materials can be considered. The corrugated waveguide is assumed to be illuminated from the input region by the fundamental $\mathrm{TE}_{0}$ or $\mathrm{TM}_{0}$ mode. The wavelength in vacuum of the incident mode is denoted $\lambda\left(k_{0}=2 \pi / \lambda\right)$.

For analysis of such waveguide geometries with RCWA, we introduce an artificial periodization along the $x$ (vertical) coordinate, thus virtually replacing the actual waveguide isolated in space with a periodic waveguide structure. We denote by $w$ the period (or a computational box) in the $x$ direction. With this periodization, we can apply RCWA to solve the diffraction problem. For the electromagnetic solution of the periodic waveguide geometry and that of the isolated waveguide structures to be identical, two modifications of RCWA have to be incorporated:

(1) Two absorbers with thicknesses $L_{c}$ and $L_{s}$ near $x=w / 2$ and $-w / 2$, respectively, are included, as shown in Fig. 1. To satisfy the outgoing and ingoing wave conditions these absorbers (a) absorb the light scattered by the corrugation, thus making electromagnetically independent the neighboring waveguides of the periodic waveguide structure, and (b) do not reflect the scattered light, thus avoiding electromagnetic contamination inside a single period. In this Letter we use a simple approach, in which the absorbers are composed of layers with complex refractive-index distributions that vary linearly from $n_{s}-0 i$ to $n_{s}-1 i$ for the substrate absorber and from $n_{c}-0 i$ to $n_{c}-1 i$ for the cover absorber. With modification (1) of the index distribution profile, the waveguide modes and their effective indices in all the layers are computed as eigenvectors and eigenvalues with the standard RCWA algorithm. Note that the electromagnetic field is looked for as a purely periodic function of the $x$ coordinate and not as a pseudo-periodic one as in standard grating theory. We obtain this function by setting the angle of incidence equal to zero in a standard RCWA code ( $\theta=0$ in Refs. 1 and 3). Using an S-matrix algorithm as we did in our simulations, the amplitudes of the modes propagating backward (in the $-z$ direction) and forward (in the $z$ direction) at the $z=0$ and $z=h$ planes, respectively, are related through

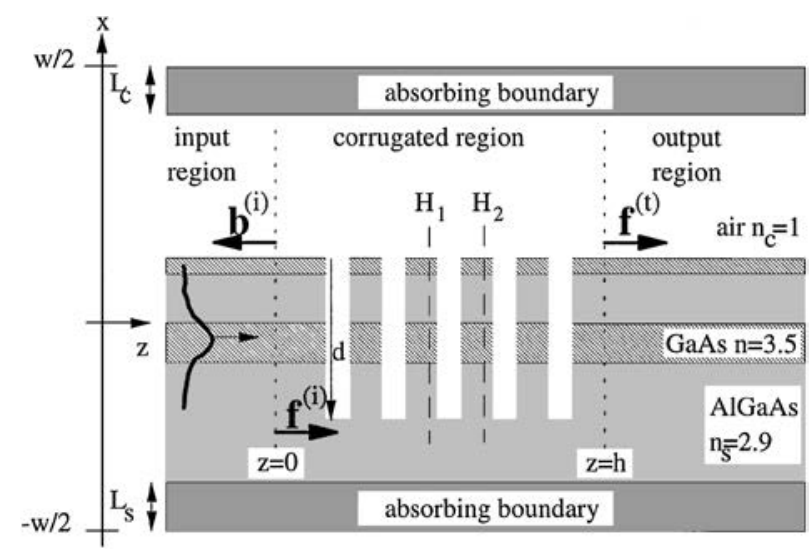

Fig. 1. Waveguide geometry: The GaAs (refractive index, 3.5) cap, the GaAlAs (refractive index, 2.9) undercap, and the GaAs core are 100, 300, and $240 \mathrm{~nm}$ thick, respectively. The groove width is $80 \mathrm{~nm}$, and the Bragg grating period is $500 \mathrm{~nm}$. $L_{c}$ and $L_{s}$ are the absorber thicknesses, and $d$ is the groove depth. 


$$
\left[\begin{array}{l}
\mathbf{b}^{(i)} \\
\mathbf{f}^{(t)}
\end{array}\right]=\mathbf{S}\left[\begin{array}{c}
\mathbf{0} \\
\mathbf{f}^{(i)}
\end{array}\right]
$$

where $\mathbf{b}^{(i)}\left[\mathbf{f}^{(i)}\right]$ are column vectors whose elements are the amplitudes of the modes propagating backward (forward) at the plane $z=0$. Similarly, $\mathbf{f}^{(t)}$ is composed of the amplitudes of the modes propagating forward at the plane $z=h$. The zero vector on the right-hand side of Eq. (1) expresses the fact that no light is impinging on the corrugated waveguide from the output region $\left[\mathbf{b}^{(t)}=\mathbf{0}\right]$. For the specific waveguide geometry of Fig. 1, the $\mathbf{S}$-matrix computation involves propagation through 11 layers.

(2) With RCWA and most grating theories, a Rayleigh expansion is used to describe the electromagnetic field in the input and output homogeneous regions. In the present case the field expansions in the input and output regions are periodic Floquet expansions like those for all the layers of the corrugated region. Assuming that the corrugated waveguide is illuminated by the fundamental $\mathrm{TE}_{0}$ or $\mathrm{TM}_{0}$ mode with a unitary amplitude, all components of vector $\mathbf{f}^{(i)}$ in Eq. (1) are zero, except the $p$ th component $f_{p}^{(i)}$, which is equal to $1, p$ being the eigenmatrix-column number that corresponds to the fundamental mode. Using Eq. (1), we then compute the amplitudes of the backward- and forward-propagating modes. If we define the reflected intensity $R$ by the amount of light that is backreflected into the fundamental input mode, $R$ is simply equal to $\left|b_{p}^{(i)}\right|^{2}$. Similarly, the transmitted intensity $T$ defined by the amount of the light transmitted into the fundamental output mode is defined by the ratio between the time-averaged Poynting vector $z$ components of the fundamental output mode and that of the fundamental input mode. $T$ is simply $\left|f_{p}^{(t)}\right|^{2}$ for the waveguide geometry of Fig. 1, since the input and output regions are identical. This second modification concerns only the treatment of the first and last interfaces at $z=0$ and $z=h$, respectively. The modification is rather small, and it even simplifies the RCWA code, since the same field expansions are used for all regions. For instance, computation of matrices $\mathbf{Y}_{\mathrm{I}}$ and $\mathbf{Y}_{\mathrm{II}}$ in Eqs. (21) and (24) of Ref. 1 is no longer needed. The programming effort needed to incorporate modifications (1) and (2) into a grating RCWA code equipped with the $\mathbf{S}$-matrix algorithm is rather minimal.

The solid curves in Fig. 2 represent the reflected and the transmitted intensities for the geometry of Fig. 1 as a function of etching depth $d$. The quantity $1-R-T$ represents the amount of scattered light. To assess the accuracy of the computation we also implemented a well-established numerical method in waveguide theory, the method of lines ${ }^{5}$ (MOL). The MOL numerical values are shown as pluses in Fig. 2. They agree well with the RCWA computation values: The maximum deviations between the MOL and the RCWA results are $0.23 \%$ for $T$ and $0.94 \%$ for $R$. The solid curves in Fig. 3 illustrate the convergence performance of the RCWA approach, for $d=1000 \mathrm{~nm}$ and for two periods, $w=3 \lambda$ and $w=6 \lambda$. Figures 3(a) and 3(b) correspond to TE and TM polarization, respectively. For comparison, the convergence rate of the MOL for a computationalwindow width of $3 \lambda$ is shown by pluses. Our MOL implementation uses a uniform discretization, the absorbing boundary conditions of Ref. 6 and the finite-difference expressions of Ref. 7, for modeling permittivity discontinuities accurately. The RCWA approach performs well; it converges even faster than the MOL approach. Typical RCWA CPU times obtained for TM polarization with a Pentium $233-\mathrm{MHz}$ processor are $4 \mathrm{~s}$ for $N=51$ and $30 \mathrm{~s}$ for $N=101$. Note that the computation requires solution of only two eigenproblems.

The Fourier approach presented in this Letter can also be used to efficiently study homogeneous problems ${ }^{8}$ in waveguide gratings. It is well established that the resonance anomalies of grating waveguide filters, the coupling lengths of grating couplers, and the stop bands of Bragg waveguide mirrors or of

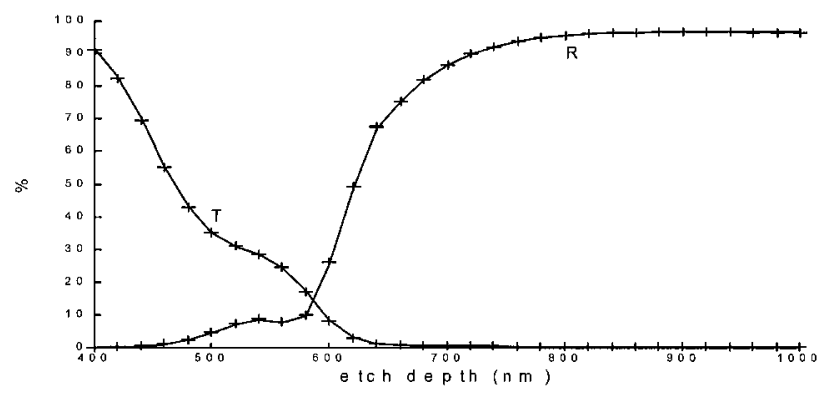

Fig. 2. Reflected and transmitted intensities for the mirror problem of Fig. 1 for TE polarization and for $\lambda=975 \mathrm{~nm}$. Solid curves, RCWA results with $N=301$ retained Fourier harmonics $\left(L_{c}=L_{s}=0.5 \lambda\right.$ and $\left.w=3 \lambda\right)$. Pluses, MOL results with 300 discretization points and a $3 \lambda$ computational-window size.
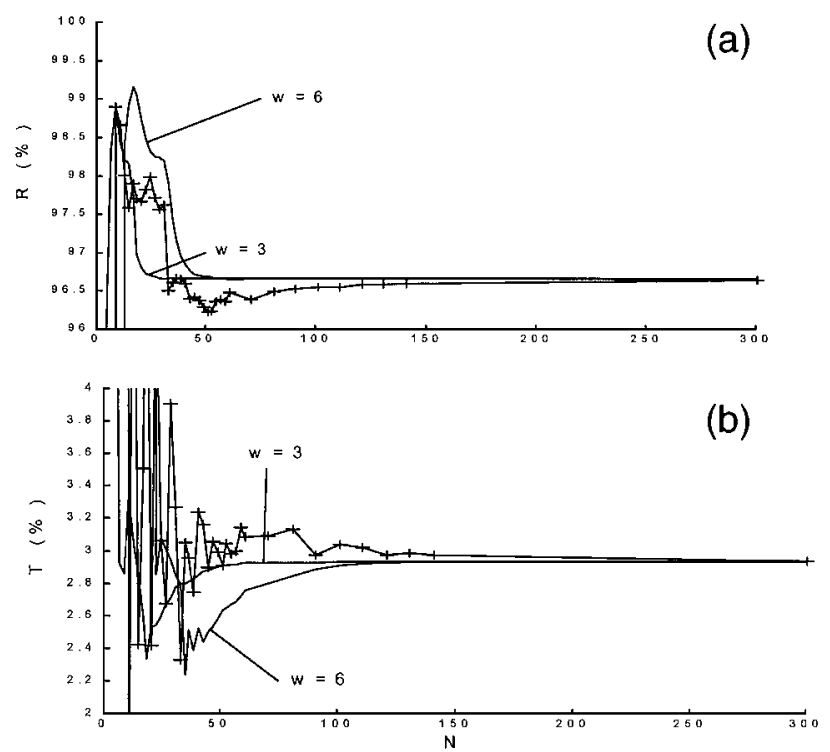

Fig. 3. Convergence performance for $d=1000 \mathrm{~nm}$. (a) Reflected intensity for TE polarization. (b) Transmitted intensity for TM polarization. Solid curves, RCWA results for $w=3 \lambda, 6 \lambda$. Pluses, MOL results for a $3 \lambda$ computational-window size. 
Table 1. $n_{\text {eff }}$ Values Obtained with $L_{c}=L_{s}=1 \lambda^{a}$

\begin{tabular}{cccc}
\hline & CPU & & \\
$N$ & Time $(\mathrm{s})$ & $w=6 \lambda$ & $w=8 \lambda$ \\
\hline TE mode & & & \\
21 & 0.7 & $0.015 i+1.577$ & $0.006 i+1.571$ \\
41 & 3.3 & $0.015 i+1.582$ & $0.014 i+1.581$ \\
61 & 10 & $0.015 i+1.582$ & $0.014 i+1.582$ \\
81 & 24 & $0.015 i+1.582$ & $0.014 i+1.582$ \\
TM mode & & & \\
21 & 0.8 & $0.0045 i+1.605$ & $0.0026 i+1.595$ \\
41 & 3.9 & $0.0044 i+1.608$ & $0.0142 i+1.608$ \\
61 & 12 & $0.0044 i+1.609$ & $0.0043 i+1.609$ \\
81 & 28 & $0.0044 i+1.609$ & $0.0044 i+1.609$ \\
\hline
\end{tabular}

${ }^{a}$ The TE and TM modes obtained in Ref. 9 are $0.014 i+1.583$ and $0.0047 i+1.609$, respectively.

surface-wave plasmons can be studied through the complex poles of the determinant of a scattering matrix that relates the diffracted and incidence waves; see, e.g., Ref. 9, and references therein. In general, the computation of the poles (or, equivalently, the effective index $n_{\text {eff }}$ of the leaky wave) requires that we find the complex root of the determinant. The amount of computation that one needs to evaluate the determinant is approximately that required for solution of the inhomogeneous grating problem. ${ }^{9}$ However, the root search involves many iterative calculations of the determinant. With the present approach, the computation of $n_{\text {eff }}$ does not require any iteration. This computation consists of solving an eigenproblem. Let us consider an arbitrary one-dimensional grating waveguide with a period $\Lambda$ along the $z$ direction. The waveguide mirror of Fig. 1, with an infinite extent along the $z$ direction, is one possible example. Let us also consider two arbitrary planes, $\mathrm{H}_{1}$ and $\mathrm{H}_{2}$, as shown in Fig. 1, located at coordinates $z=z_{1}$ and $z=z_{2}$, respectively. We assume that $z_{2}-z_{1}=\Lambda$. With straightforward notation, the amplitudes of the backward- and forward-propagating modes at planes $\mathrm{H}_{1}$ and $\mathrm{H}_{2}$ are related by

$$
\left[\begin{array}{l}
\mathbf{b}^{(2)} \\
\mathbf{f}^{(2)}
\end{array}\right]=\mathbf{T}\left[\begin{array}{l}
\mathbf{b}^{(1)} \\
\mathbf{f}^{(1)}
\end{array}\right],
$$

where the $\mathbf{T}$ matrix is simply the so-called transmission matrix. ${ }^{2}$ For binary lamellar gratings, the T-matrix computation requires that we solve two eigenproblems and invert two eigenvector matrices. The effective index $n_{\text {eff }}$ is related to the eigenvalue $\mu$ of matrix $\mathbf{T}$ by $\mu=\exp \left(-j k_{0} n_{\text {eff }} \Lambda\right)$. The leaky mode is the corresponding eigenvector.

To test the efficiency of the approach we consider the lamellar grating waveguide problem studied by Chang et $a l .{ }^{9}$ Table 1 shows the $n_{\text {eff }}$ values obtained with $L_{c}=L_{s}=1 \lambda$ for several values of $w$ and $N$ and for a $1 \lambda$ groove depth $\left(t_{g}=\lambda\right.$ in Table III of Ref. 9). Basically, excellent agreement with the values obtained by Chang et al. is found, the largest difference between the two sets of results being less than 0.001. CPU times obtained with a personal computer equipped with a Pentium 166-MHz processor are given in Table 1.

The numerical results reported here are encouraging. However, the artificial periodization used in this Letter (equivalently, the use of Fourier series in waveguide theory) is far from being on solid mathematical ground, and much work is required for the present approach to be validated. For that purpose, better absorbers have to be designed. From the point of view of waveguide theory, the present Fourier method belongs to the general class of mode-matching methods; it improves on previous work, ${ }^{10,11}$ which relied on the use of Fourier analysis. From the point of view of grating theory, the present method extends the domain of applicability of RCWA. A similar approach has been used for electromagnetic analysis of distributed Bragg reflectors. ${ }^{12}$ We believe that other grating theories may benefit from this work.

The authors thank J. P. Hugonin and P. Chavel for fruitful discussions. This work was supported under the program Microsystèmes. P. Lalanne's e-mail address is philippe.lalanne@iota.u-psud.fr.

\section{References}

1. M. G. Moharam, E. B. Grann, D. A. Pommet, and T. K. Gaylord, J. Opt. Soc. Am. A 12, 1068 (1995).

2. L. Li, J. Opt. Soc. Am. A 13, 1024 (1996).

3. Ph. Lalanne and G. M. Morris, J. Opt. Soc. Am. A 13, 779 (1996).

4. T. F. Krauss, O. Painter, A. Scherer, J. S. Roberts, and R. M. De La Rue, Opt. Eng. 37, 1143 (1998).

5. Session C09 of the 1998 Progress in Electromagnetics Research Symposium, Nantes, France, July 13-17, 1998.

6. J. Gerdes, B. Lunitz, D. Benish, and R. Pregla, Electron. Lett. 28, 1013 (1992).

7. S. F. Helfert and R. Pregla, J. Lightwave Technol. 14, 2414 (1996).

8. E. Popov, Prog. Opt. 31, 141 (1993).

9. K. C. Chang, V. Shah, and T. Tamir, J. Opt. Soc. Am. 70, 804 (1980).

10. C. H. Henry and B. H. Verbeek, J. Lightwave Technol. 7, 308 (1989).

11. J. Rodriguez, R. D. Crespo, S. Fernandez, J. Pandavenes, J. Olivares, S. Carrasco, I. Ibanez, and J. M. Virgos, Opt. Eng. 38, 1896 (1999).

12. P. Vahihama and J. Turunen, in Diffractive Optics and Micro-Optics, Vol. 10 of 1998 OSA Technical Digest Series (Optical Society of America, Washington, D.C., 1998), p. 69. 